\title{
Emergent Anagram and Vocal Spelling via Stimulus Equivalence in Japanese Children With Intellectual Disabilities
}

\author{
Takayuki TANJI ${ }^{1, *}$ and Fumiyuki Noro ${ }^{2}$ \\ ${ }^{1}$ Graduate School of Education, Okayama University, Japan \\ ${ }^{2}$ Faculty of Human Sciences, University of Tsukuba, Japan
}

\begin{abstract}
The present study examined the effects of anagram and vocal spelling instruction on emergent both spelling for untaught words in two Japanese participants with intellectual disabilities. A multiple probe design was employed across three Sets. The participants were taught to spell 2-letter words with Set 1 as anagram and vocal spelling, and then were tested the responses for untaught words with Sets 2 and 3. The words that the participants could read were used in those Sets, however they could not construct the words. A participant showed emergent anagram and vocal spelling responses in untaught Sets 2 and 3 immediately following Set 1 instruction. However, another participant who had no spelling learning history did not show emergent spelling immediately and needed additional instruction. The results are discussed in terms of stimulus equivalence, participant's history of reinforced exemplars for spelling, mediating effects of vocal spelling and textual repertoire, and development of language and moraic segmentation repertoire.
\end{abstract}

Key Words: anagram spelling, vocal spelling, stimulus equivalence, intellectual disabilities, textual

\section{Introduction}

In behavioral analytic approach, previous research have analyzed emergence of untrained reading and spelling (e.g., anagram spelling, written spelling) behavior using stimulus equivalence paradigm. Stimulus equivalence paradigm was applied to teach rudimentary reading and spelling repertoire to individuals with intellectual disabilities (Mackay, 1985; Sidman, 1971). Stimulus equivalence is often established through the matching-to-sample (MTS) procedure. When A1 (e.g., the spoken word $/ \mathrm{dog} /$ ) is presented as a sample stimulus, the participant is required to select $\mathrm{B} 1$ (e.g., picture of a $\operatorname{dog}$ ), and when A2 (e.g., the spoken word /cat/) is the sample, the participant is required to select B2 (e.g., picture of a cat). Furthermore, when A1 is presented as a sam-

\footnotetext{
* Corresponding Author

Mailing Address: 3-1-1 Tsushima-naka, Kita-ku, Okayama-shi, Okayama 700-8530, Japan

E-mail Address: tanji@okayama-u.ac.jp

Received June 16, 2016, Accepted February 18, 2017

DOI: $10.6033 /$ specialeducation.6.33
}

ple, the participant is required to select $\mathrm{C} 1$ (e.g., the printed word dog), and when $\mathrm{A} 2$ is the sample, the participant is required to select C2 (e.g., the printed word cat). MTS procedures may generate derived stimulus relations, i.e., when $\mathrm{B} 1$ is presented as a sample, the participant without training may select $\mathrm{C} 1$, and when $\mathrm{B} 2$ is presented as a sample, the participant without training may select $\mathrm{C} 2$. Furthermore, when $\mathrm{C} 1$ is the sample, the participant may select $\mathrm{B} 1$, and when $\mathrm{C} 2$ is the sample, the participant may select B2. Such derived stimulus relations are called stimulus equivalence (Sidman \& Tailby, 1982).

Mackay (1985) reported that constructed-response matching-to-sample (CRMTS) procedure is effective for teaching rudimentary spelling behaviors in individuals with intellectual disabilities. Mackay taught students with intellectual disabilities to name color patches and construct the appropriate color words, when the patches were shown, using movable letters. For example, if the color patch "red" was presented as the sample stimulus and the letters "r", "e", and "d" were presented as the comparison stimuli, the students were required to select " $r$ ", "e", and "d" from a choice of letters in the CRMTS task. After 
the CRMTS instruction, the students could match printed words to color patches and color patches to printed words and also read printed words without direct instructions. CRMTS instruction produced stimulus equivalence among the colors, printed names, and spoken names. These findings suggest that stimulus equivalence is a behavioral analytic paradigm including both reading and spelling behaviors.

Stimulus equivalence paradigm was applied to teach reading hiragana for children with reading difficulty. MTS procedure was used for reading hiragana words instruction (Kikuchi, 1985; Tsurumaki, 1995). CRMTS procedure was used for reading single hiragana letters through the learning one to one correspondence morae and hiragana-letters in a word (Morita, Nakayama, Sato, \& Maekawa, 1997; Sugasawara \& Yamamoto, 2007). However, few studies demonstrated that CRMTS were effective for emergent anagram spelling (Sakamoto, Hiruma, Hosokawa, Imanaka, \& Maekawa, 2004; Tanji, Takahashi, \& Noro, 2013).

In a recent research on behavioral instructional protocols for emergent spelling behaviors, the relationships between emergence of taking dictation (i.e., written spelling controlled by verbal stimulus) and vocal spelling (i.e., spelling in vocal form) has been discussed. de Souza and Rehfeldt (2013) examined the emergence of vocal spelling following dictation-taking instruction for three men with intellectual disabilities whose ages ranged from 18 to 20. Their Intelligence Quotient (IQ) scores ranged from 61 to 72 on the WAIS-IV, and they could spell simple three-letter words before the experiment. In the experiment, participants were tested vocal spelling performances (e.g., the participant said "e, x, p, a, n, d" after the experimenter said "Spell expand") following the dictation-taking instruction (e.g., the participant wrote "expand" after the experimenter said, "Write expand") with nine 6- to 11- letter words. Results showed that all participants' untrained vocal spelling performances improved following dictationtaking instruction alone. Thus, the results suggest that written spelling corresponding to dictated words instruction improved vocal spelling performances. The positive results may be due to participants' emission of covert textual (i.e., reading out loud controlled by verbal units in written stimulus) responses in the dictation-taking task. That is, the correct written spelling may have allowed the participants to say the letters of the word correctly, and then would influence the positive effects on their vocal spelling.

Although previous studies suggest that nontargeted vocal spelling behavior emerged after written spelling instruction (de Souza \& Rehfeldt, 2013), it is necessary for participants who have poor learning histories of spelling to instruct both written and vocal spelling (Greer, Yaun, \& Gautreaux, 2005). Greer et al. tested the effect of multiple exemplar instruction (MEI) on emergence of vocal to written and written to vocal spelling responses, for three sets of five 2- to 5- letter words in five- to six-year-old kindergarteners with language delays. Their IQ scores ranged from 59 to 76 on the Stanford Binet Intelligence Scale, and they could write letters $\mathrm{A}-\mathrm{Z}$ when dictated, and some words, and read 15 to 200 words. First, participants were taught to spell words in Set 1 in either vocal or written form, and then were tested on untaught words. Next, participants were taught words in Set 2 using multiple-exemplar instruction (MEI) (i.e., instruction for alternating responses) and were tested on untaught words for Set 1 again. Finally, participants were taught to spell words in Set 3 in either vocal or written form and were tested on untaught words. Results showed that all participants' spelling performances on untaught words were low in accuracy before MEI instruction, but improved following MEI.

The finding of Greer et al. differs from that of de Souza and Rehfeldt (2013). de Souza and Rehfeldt suggested that the difference of participants' history of reinforced exemplars for written and vocal spelling and participants' textual repertories between these studies resulted in the different findings. In de Souza and Rehfeldt study, the participants had a longer history of reinforced exemplars for writing and spelling and more sophisticated textual repertories (e.g., reading fluently the syllable units of words) than those of Greer et al.

Previous research examined the relationship between written spelling and vocal spelling, however there are few studies that examined whether emergent relation between anagram spelling and vocal spelling are demonstrated in young children with intellectual disabilities. Dairoku (1996) indicated that some Japanese individuals with intellectual disabilities can read most single hiragana letters but cannot recognize a string of letters as a word, and others have difficulty in shifting alphabet reading phase (i.e., 
reading hiragana with phonological awareness) from logographic reading phase (i.e., reading hiragana with non-phonological procedures). Amano (1977) and Dairoku (1996) suggested that it is important for these individuals to raise their phonological awareness. Dairoku (1996) suggested that anagram spelling instruction could facilitate phonological awareness (e.g., "moraic segmentation" and "moraic isolation"). However, there is no study that empirically examined the effect that anagram spelling instruction facilitates phonological awareness. The present study assumed that vocal spelling in Japanese words is similar to moraic segmentation (e.g., saying each mora, /u/ and $/ \mathrm{shi} /$, in the presence of the picture of “うし"). If anagram spelling instruction improves vocal spelling performances in young children with intellectual disabilities, their anagram and vocal spelling performances in untaught readable words also would be improved (Tanji et al., 2013).

The present study examined the effects of anagram spelling instruction with multiple exemplars on emergent vocal and anagram spelling for untaught words in two young Japanese children with developmental delay. It seemed that they were on the logographic reading phase because they could not understand the meaning of words and match correct picture to printed word although they could read some words.

\section{Method}

\section{Participants}

The first participant, Ken (pseudonyms are used in this article for protection of privacy of the participants) was a seven-year-old elementary first-grade male pupil. He was diagnosed with intellectual disabilities. He was enrolled in a public special needs school. On the Tanaka-Binet Intelligence Scale fifth edition (Tanaka Institute for Educational Research, 2003), his IQ was 67, and his mental age was 5 years and 0 months. His vocabulary age was 4 years and 5 months on the Picture Vocabulary Test-Revised (PVT-R; Ueno, Nagoshi, \& Konuki, 2008). He could read half of the 46 single hiragana letters, and read individual letters of 2-letter words but could not match pictures to printed words. When the experimenter conducted the printed word-picture matching task, he could sound out individual letters of printed 2-letter words but could not match the correct pic- tures to the printed words. He had a few experience of learning anagram spelling (e.g., constructing printed words using cards of printed single hiragana letters to dictated words) in his school. He could not write any hiragana letters.

The second participant, Taro (pseudonyms are used) was a kindergarten boy. He was a 5-year-old and diagnosed with autism spectrum disorders. $\mathrm{He}$ was enrolled in a public kindergarten. On the Kyoto Scale of Psychological Development 2001 (Ikuzawa, Matsushita, \& Nakase, 2002), his total developmental age was 2 years and 10 months, physical-motion age was more than 4 years, cognitive-adaptation age was 3 years and 2 months, and verbal-social age was 2 years and 5 months. His vocabulary age was less than 3 years on the PVT-R (Ueno et al., 2008). He could make two-word and three-word utterances and could reply to some simple questions (e.g., "What's your name?" or "How old are you?"). However, his speech was often unintelligible. He could not correctly pronounce some words (e.g., $k a-s a \rightarrow k a-t y a$, $k a-m e \rightarrow t a-m e$, and $t a-k o \rightarrow t a-t o)$. He could read most of the 46 basic single hiragana letters, and read individual letters of 2-letter words but could not match the correct pictures to the printed words. When the experimenter conducted the printed word-picture matching task, he could sound out individual letters of the printed 2-letter words but could not match the correct pictures to the printed words. Before this study, he showed response when pronouncing a word slowly while clapping his hands (e.g., He segmented the word "tyo-da-i" into "tyo" and "dai" in a way that pronounces "tyo" while making the first clap and "dai" while making the second clap). He had no experience in spelling. His mother stated in an interview that he had refused to engage in anagram spelling tasks and displayed problem behaviors (e.g., throw and tear the teaching materials) when a clinician conducted anagram spelling to pictures task.

The first author explained the content of the study to their mothers in written form, and received their approval. This study received approval of the ethical committee at Tsukuba University, Graduate School of Comprehensive Human Sciences.

\section{Settings}

Ken's instruction and test sessions were implemented in a quiet room in his school. He sat in front of a 15-inch touch-sensitive desktop monitor and a 
personal computer. The present study called a unit of instruction and test in a session as a "block". One block consisted of four trials. A word was presented in a trial, and four words in a set were presented once in a block. Most of the time, three blocks were held in a session. A session was held once a week; each session lasted for 10 minutes.

Taro's instruction and test sessions were implemented in a therapy room in a university. All spelling tasks were conducted on the tabletop in a one to one instruction setting by the experimenter. Taro was restless, and it was difficult to engage in academic tasks alone. It was necessary for him to work with an experimenter. The computerized anagram spelling task required him to work calmly to accomplish this task. Thus, authors of the present study judged that it was not suitable for Taro to take the sessions in computerized format. One block consisted of four trials. Most of the time, three blocks were implemented in a session. A session was held once a week; each session lasted for 15 minutes including break time.

\section{Materials}

For Ken, the present study used a 15 -inch touchsensitive desktop monitor and a personal computer to conduct the test and instruction. On the touchsensitive monitor, auditory and visual stimuli were presented (see Tanji et al., 2013). Auditory stimuli were dictated words, and three types of visual stimuli were used; pictures, printed single letters, and printed words. An auditory stimulus was presented as a sample stimulus in the anagram spelling corresponding to dictated words task. A picture was presented as a sample stimulus in the anagram spelling corresponding to pictures task. A printed word was presented as a sample stimulus in the anagram spelling corresponding to printed words task. In these spelling tasks, four printed single letters were presented as comparison stimuli on a letter-choice pool to construct words.

For Taro, the test and instructions were conducted using a whiteboard (board size, $30 \mathrm{~cm}$ by $50 \mathrm{~cm}$ ). Visual sample stimuli (e.g., printed words or pictures) were presented at the top of the whiteboard. At the bottom of it, comparison stimuli (e.g., printed single letters) were presented. A construction area was placed to move letters below the sample stimuli to form the words. All visual stimuli (e.g., pictures, printed words, and printed single letters) were on laminated, $5 \mathrm{~cm}$ cards. Auditory stimuli (i.e., dictated words) were presented as sample stimuli by the experimenter.

\section{Stimuli}

The present study used twelve 2-letter words for Ken. These words were divided into three sets; Set 1: “引し” (u-shi; cow), “うま” (u-ma; horse), “くし” (kushi; comb), “くま” (ku-ma; bear); Set 2: “すし” (su-shi; sushi), “すな” (su-na; sand), “はし” (ha-shi; chopstick), “はな” (ha-na; flower); Set 3: “あお” (a-o; blue), “あめ” (a-me; candy), “かお” (ka-o; face), “かめ” (ka-me; turtle). The present study used the words that Ken could read and name aloud but his teacher did not target in his school. To ensure that he could read the printed words and name the pictures, the experimenter presented each of them to him and instructed if he did not respond correctly.

For Taro, the present study also used twelve words that he could read and name aloud. Three 2-letter word sets were used; Set 1: “引し” (u-shi; cow), “はし” (ha-shi; chopstick), “かさ” (ka-sa; umbrella), “かに” (ka-ni; crab); Set 2: “あし” (a-shi; foot), “ほし” (ho-shi; star), “かお” (ka-o; face), “かめ” (ka-me; turtle); Set 3: “いす” (i-shu; chair), “くつ” (ku-tsu; shoes), “たこ” (tako; octopus), “子小” (fu-ne; ship). The word set was different from Ken's because Taro did not know or could not name some of the words. Also, authors of the present study thought that it was difficult for him to discriminate morae sound of words if the present study used many words with common morae (e.g., “引 し”, “うま”, “くし”, and “くま”).

\section{Procedure}

The present study conducted three anagram spelling tasks: anagram spelling corresponding to printed words, pictures, and dictated words. These anagram spelling tasks required construction of printed words through sequential choice of single letters. In both the anagram spelling to pictures task and anagram spelling to dictated words task, he was also required to spell in point-to-point vocal form corresponding to dictated words or pictures (i.e., vocal spelling).

A non-concurrent multiple probe design across word sets was employed. The present study did not implement concurrent probes during the instruction. After the instruction with a set was finished, the experimenter implemented probes in taught and untaught sets. 
Baseline and probe. The present study implemented the baseline phase before the instruction with Set 1 . First, the present study implemented the anagram spelling to printed word task. A printed word as a sample stimulus was presented, and four printed single letters were presented as comparison stimuli. The experimenter said "Look at the printed word and read it, and make the same word by selecting letters from the list". The participants were required to match the printed words through the sequential choice of single letters after reading the words. In this task, the experimenter evaluated only anagram spelling performance.

Next, the anagram spelling to dictated words task was assessed. This task was the same as the anagram spelling to printed words task except the sample stimuli were dictated words. When a sample stimulus was presented, the experimenter said "Listen to the word and remember it, and make the same word by selecting the letters from the list". In this task, the experimenter evaluated vocal and anagram spelling performances. The participants were required to not only construct the words to the sample stimuli (i.e., anagram spelling) but also sound out corresponding to each mora of words (i.e., vocal spelling). For example, if the dictated word, $k a-s a$, was presented, the present study evaluated whether he sounded out the first mora, $k a$, before choosing the letter, “か”, and the second mora, $s a$, before choosing the letter, “さ”. No feedback was given to anagram and vocal spelling performances. Probes were implemented after the instruction with each set, in the same procedure as in the baseline.

Finally, the anagram spelling to pictures task was assessed. This task was the same as the anagram spelling to dictated words task except the sample stimuli were pictures. When a sample was presented, the experimenter said "Look at the picture and think of the word that the picture represents, and make the same word by selecting the letters from the list". The present study evaluated both spelling performances as conducted in the anagram spelling to dictated words task.

Correct responses were defined as responses that correspond point to point to conventional spelling; incorrect responses were defined as no response or responses that do not correspond point to point. Feedback on whether the responses were correct or incorrect was not given. The criterion performance was to have at least seven out of eight correct spelling responses for two consecutive blocks in each task.

For Taro, the procedures were the same as Ken except for the use of tabletop format in presentation of stimuli and the use of rewards. Sample and comparison stimuli were not automatically presented by the computer but manually by the experimenter. Also, the present study employed a token economy system for Taro to maintain his motivation to engage in the tests. When a trial was over, he was given a sticker as a token. If Taro saved eight stickers, he was able to trade them with a piece of his favorite snack. Probes were conducted after each of the instruction for Sets 1, 2, and 3 was completed.

Instruction. The present study instructed all three anagram spelling tasks. The instructional phases were composed of two steps.

In Step 1, Ken was taught to construct words through the sequential choice of letters corresponding to printed words. In the anagram spelling to printed words task, the experimenter read each printed letter of the sample printed words before he constructed the words (i.e., When the sample printed word, “うし”, was presented, the experimenter sounded out the first mora, $u$, before the participant selected the single letter, “ $う$ ”, and sounded out the second mora, shi, before the participant selected the single letter, "L"). Ken was required to select the correct single letters attending to the printed letters of the sample words and morae sound of them given by the experimenter. The present study assessed only anagram spelling performance. If Ken showed correct anagram spelling responses, verbal praise by the experimenter and pictures of his favorite anime characters were displayed on the computer. If he showed incorrect responses, sample stimuli and the comparison stimuli disappeared and they were presented again, and retrials were conducted until he provided correct responses. A criterion performance was to have at least seven out of eight correct spelling responses for two consecutive blocks. Instruction continued until the criterion was met; however, the experimenter might carry out an extra training after the achievement.

However, the present study conducted a step-bystep sequence of instruction for Taro in the anagram spelling to printed words task with Set 1 because he had no instructional history with spelling. Step 1 was divided into four steps. In Step 1-1, the printed letters 
to construct the word were sequentially shown. After Taro read the printed word that was presented as a sample, the first letter as a comparison was presented, and he was required to choose it. Then, the second letter was shown, and he was required to choose it as well. It was an errorless learning. In Step 1-2, two comparisons were simultaneously shown. Taro was required to choose the letters that corresponded to the letter sequence of the word as a sample. Step 1-3 was the same as step 1-2, except that a wrong letter was presented: In step 1-4 was the same as step 1-3, except that four comparison stimuli were presented. During instruction phases, the present study continued to employ the reward system. Feedback on correct anagram spelling was given as verbal praise, tickling, and a sticker by the experimenter. When anagram spelling were incorrect, the experimenter said "Try again" to him. Taro was required to engage in a re-trial after the experimenter returned the stimuli. Instruction continued until he responded correctly in at least seven out of eight trials for two consecutive blocks. When the criterion was met, the next task was implemented. The present study omitted explicit instruction when the criterion was met during probes.

In Step 2, the experimenter implemented the anagram spelling to dictated words task and the anagram spelling to pictures task in turn. The participants were taught to construct words through the sequential choice of letters corresponding to dictated words and pictures. If there were no response or vocal spelling was incorrect, the experimenter presented correct vocal spelling before the participants selected single letters. The participants were required to imitate correct vocal spelling responses given by the experimenter. If the participants showed correct vocal spelling responses, verbal praise was given by the experimenter. Feedback to correct or incorrect anagram spelling responses were the same as in the spelling to printed words task. A criterion performance was to have at least seven out of eight correct vocal and anagram spelling responses to dictated words and pictures for two consecutive blocks. Instruction continued until the criterion was met; however, the experimenter might carry out an extra training after the achievement. Ken was not taught for Sets 2 and 3 because both spelling performances met the criterion in the probe after instruction for Set 1.

\section{Reliability}

The first author and a trained graduate research assistant evaluated whether the participants' anagram and vocal spelling responses were correct or incorrect. One-third of the total trials in all tasks were scored by the two independent observers. Ratings were collected from the video recordings of the responses. Inter-observer agreement between observers was calculated by dividing the number of agreements by the total number of agreements and disagreements and multiplying by 100 . The mean inter-observer agreement for anagram and vocal spelling for Ken was 100 and $94 \%$, and 93 and $91 \%$ for Taro.

\section{Results}

Figure 1 shows the number of correct spelling responses for Ken. During the baseline, he could not display correct anagram and vocal spelling responses. As soon as instruction for Set 1 began, he began to display correct anagram spelling performances. In Step 1, he showed perfect anagram spelling responses in the fourth session. In Step 2, he showed three or four correct vocal and anagram spelling responses in spite of the first session. In particular, his vocal spelling performance was perfect. When he looked for correct printed single letters, he uttered the morae sound of each letter (e.g., When the sample word, “う し", was presented, he uttered the first mora sound of the word, $u \ldots . . u \ldots$, looking for the printed letter “ $う$ ”). The criterion was met with the fourth block while he kept on performing three or more correct responses.

During probe, Ken showed perfect anagram spelling responses in all three anagram spelling tasks in Set 1 . He responded correctly on three or more vocal spelling in all three tasks. The criterion was met in the sixth session. In Sets 2 and 3, the number of correct responses was also high. The criterion was met in the sixth block in both Sets 2 and 3. Although Figure 1 does not show the number of correct matching of printed words to pictures, Ken came to display correct matching in all the words of the Sets after the instruction.

Figure 2 shows the number of correct spelling responses for Taro. During the baseline, he spelled very few words correctly across the three Sets. Immediately after implementing instruction in Set 1 , he gave three or more correct anagram spelling responses in almost all sessions during Step 1-1 to 


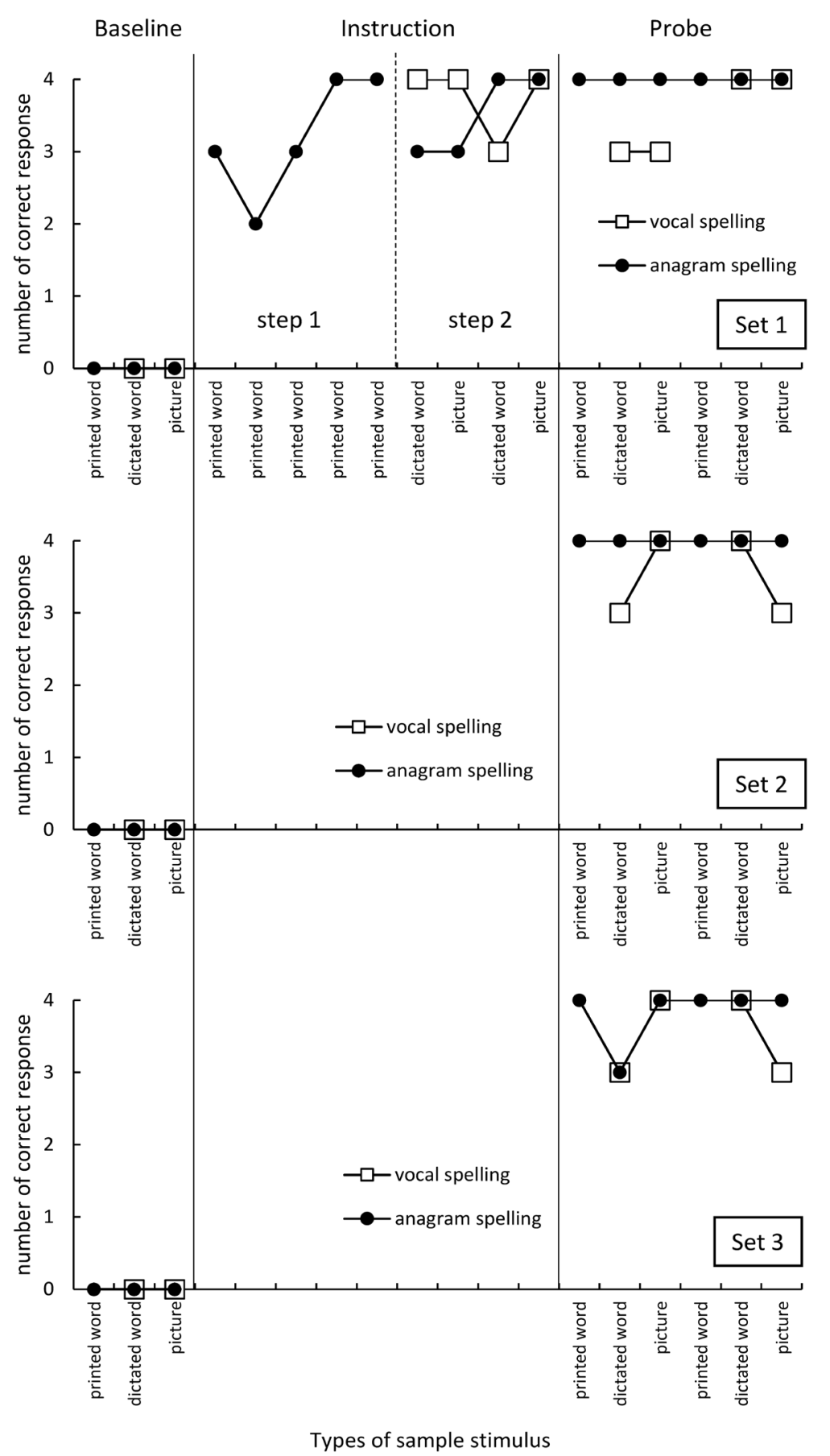

Fig. 1 Number of Correct Anagram and Vocal Spelling Responses Across Sets for Ken

1-4. During Step 2, he gave few correct anagram and vocal responses in the first two blocks. From the third block, Taro recorded 3 or more correct vocal and anagram spelling responses.

During the first probe following instruction for Set 1, Taro responded correctly to all spelling tasks in Set 1. Although the criterion was met in the anagram spelling to printed words task in Set 2, that was not met in the anagram and vocal spelling to the remaining two tasks. Taro sometimes chose printed letters without performing vocal spelling. Otherwise, he sometimes chose the wrong printed letters while performing correct vocal spelling. Immediately after implementing instruction for Set 2 , the criterion was met in spelling to dictated word and spelling to picture tasks.

During the second probe following instruction for Set 2, Taro responded correctly to all spelling tasks in 


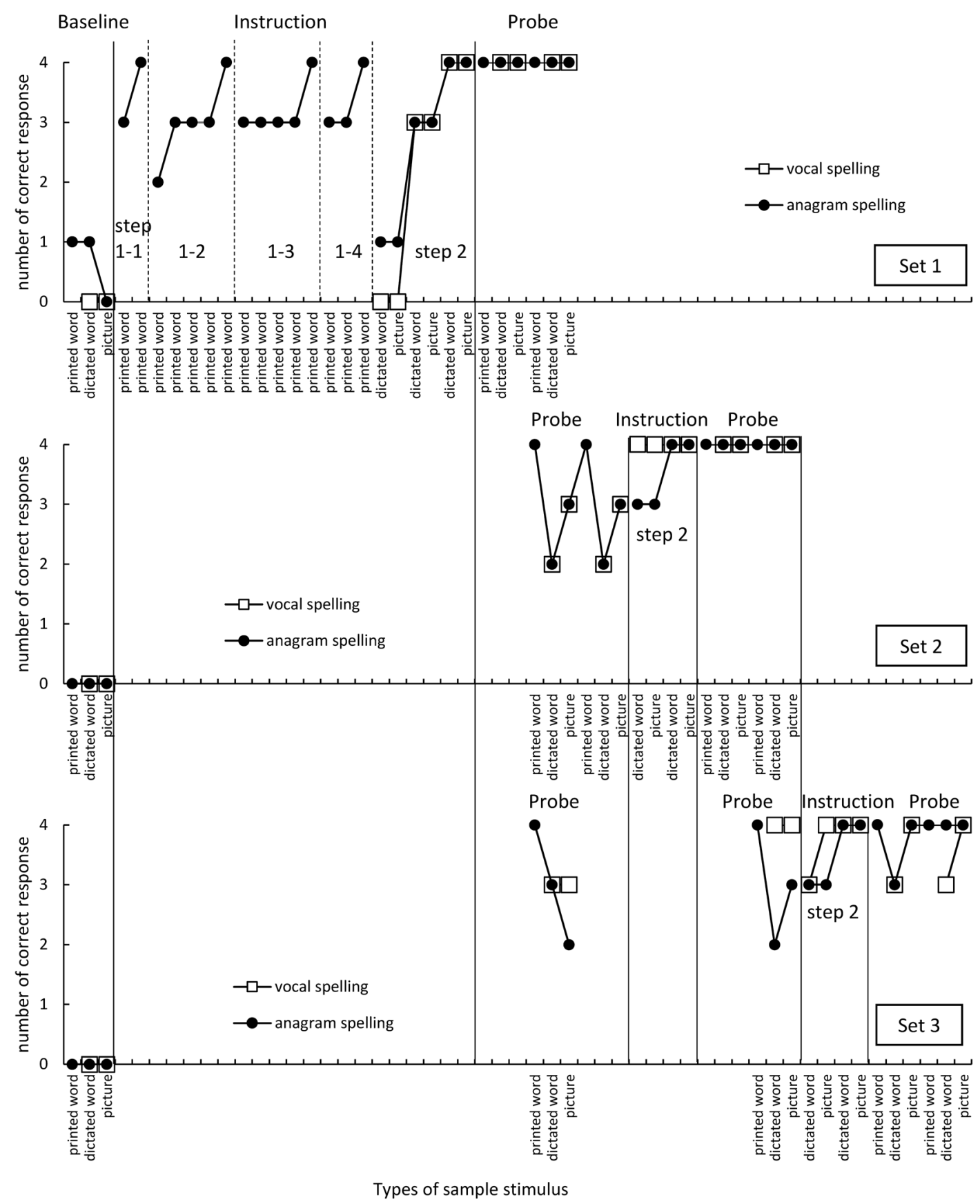

Fig. 2 Number of Correct Anagram and Vocal Spelling Responses Across Sets for Taro

Set 2. Although the criterion was met in the anagram spelling to printed words task in Set 3, that was not met in the anagram and vocal spelling to the remaining two tasks. Taro occasionally displayed incorrect anagram spelling although his vocal spelling was perfect. For example, when the word, $t a-k o$, in dictated or picture form was presented as a sample, he misspelled the word, “たい" (ta-i), and when the word, "ku-tsu", in the same manner was presented, he misspelled the word, “つく" $(t s u-k u)$. As seen in Set 2, immediately after implementing instruction for Set
3, the criterion was met in spelling to dictated word and spelling to picture tasks. During the third probe following instruction for Set 3, the criterion was met. Figure 1 does not show the number of correct matching printed words to pictures, however Taro came to display correct matching in all the words of Sets after the spelling instruction.

\section{Discussion}

First, the present study analyzed Ken's results. 


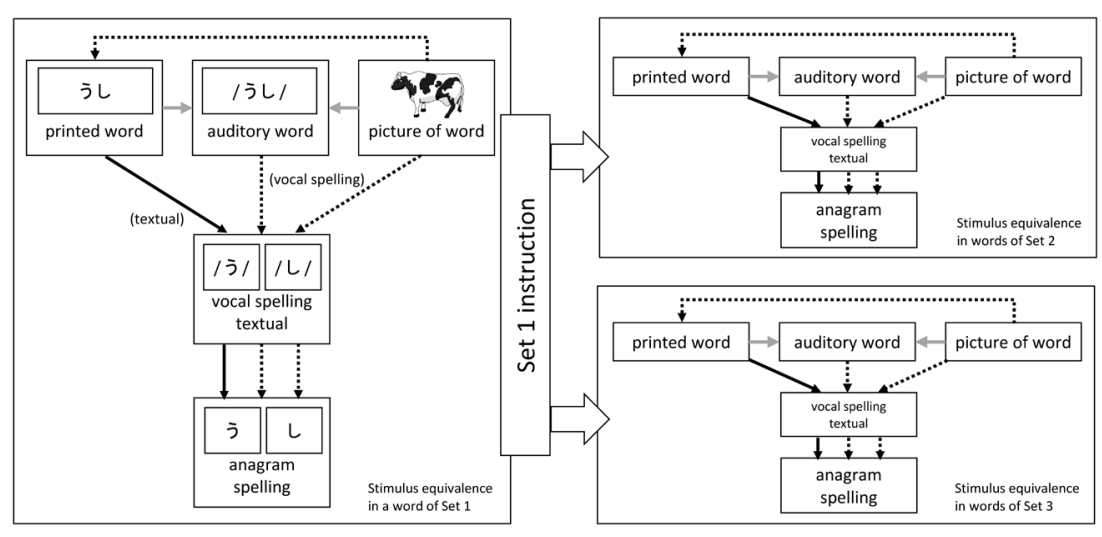

Fig. 3 Emergent Anagram and Vocal Spelling via Stimulus Equivalence in This Study

Results showed that his anagram and vocal spelling performances with untaught Sets improved following the instruction for Set 1. It assumed that both emergent spelling responses occurred following anagram instruction with Set 1.

For Set 1, the performances of anagram and vocal spelling to dictated words and pictures improved immediately after implementing the instruction in the anagram spelling to printed words task. It seemed that taught anagram and vocal spelling responses emerged in the presence of dictated words and pictures. Ken voluntarily performed vocal spelling responses in the spelling to dictated words and pictures tasks without direct instruction. Ken might have imitated the strategy of vocal spelling that the experimenter showed in the spelling to printed words task.

The present study notes below the reason why correct anagram spelling responses emerged immediately. The left side of Figure 3 illustrates the stimulus equivalence network in this study. Gray solid arrows show the relations that the participant has already learned, black solid arrows show the relations that were taught, and dashed arrows show the emerged relation. Before the instruction, it assumed that Ken learned the auditory word-printed word stimulus relations because he could read the printed words. Moreover, it assumed that Ken learned the auditory word-picture stimulus relations because he could name the pictures. Therefore, stimulus equivalent relation between auditory words and pictures of the words, and between auditory words and printed words could be established. One explanation for the results may be that the stimulus and response generalization occurred via stimulus equivalence. The learned anagram spelling responses in the presence of printed words were generalized to pictures and auditory words that are equivalent to printed words. Moreover, the improvement of anagram and vocal spelling performances in the presence of pictures would result in the emergent matching pictures to printed words performances.

Anagram spelling instruction also improved vocal spelling, and both spelling responses were generalized in untaught words. Before the present study, Ken could read individual letters of printed words with all Sets. It assumed that equivalent auditory-visual stimulus relations (i.e., printed word and auditory word relations) among each word of all Sets were established. One explanation for the results may be that the strategies of anagram and vocal spelling taught in Set 1 could be applied to the untaught words in Sets 2 and 3. In other words, the learned anagram and vocal spelling responses emerged in the other stimulus equivalent network (see the right side of Fig. $3)$. For Ken, the Set 1 instruction became adequate exemplars for generalization of both spelling performances.

This study suggests that the procedure of vocal spelling plays an important role in emergent anagram spelling. The right side of Figure 3 shows a hypothetical role of vocal spelling in emergent anagram spelling. The present data supports the idea that vocal spelling responses had a function as precursors of emitting subsequent anagram spelling. Figures 1 and 2 show that the accuracy of anagram spelling improved when correct vocal spelling occurred. Sounding out each mora of the words might make it easy to perform anagram spelling. Tanji et al. (2013) suggested that introduction of the responses to attend to each mora of the words facilitated correct anagram spelling responses. Thus, participants' mediating 
vocal spelling might serve as a discriminative function over their subsequent anagram spelling. Matos, Avanzi, and McIlvane (2006) and Hubner, Gomes, and McIlvane (2009) employed the procedure that required the four- to six-year-old kindergarten participants to sound out each syllable in anagram spelling to dictated and printed word instruction. As a result, their reading scores improved in untaught words that consisted of syllables that they could read. However, Matos et al. (2006) and Hubner et al. (2009) did not examine generalization of anagram spelling. The present study shows that anagram instruction with vocal spelling could facilitate generalization of anagram spelling.

Next, the present study analyzed Taro's results. Taro had no history of learning spelling, however his vocal and anagram spelling responses in untaught word improved after Set 1 instruction. These results suggest that this intervention brought emergent vocal and anagram spelling in an intellectual disabled child who did not have spelling repertories.

However, there were some differences between the results of Ken and Taro. Taro did not immediately show either emergent anagram or vocal spelling to dictated words and pictures in Set 1. In addition, the perfect emergent spelling in untaught Sets did not occur following Set 1 instruction. Although Ken did not require instructions in Sets 2 and 3, Taro needed the additional instruction for Sets 2 and 3.

One explanation for the results may be that Taro had no training history in anagram and vocal spelling. Emergent spelling responses through the stimulus equivalence might not immediately occur in individuals who have no training history in spelling. The results suggest that it was necessary for this type of students to have experience that reinforces their anagram and vocal spelling under various stimulus conditions to facilitate the stimulus and response generalization via stimulus equivalence. Emergent anagram and vocal spelling did not occur immediately following Set 1 instruction, however, both emergent spelling in Set 2 occurred after the criterion was met in Set 1. As Greer et al. (2005) suggested, MEI instruction was important for Taro to show emergent anagram and vocal spelling.

Second, Taro often displayed errors of anagram spelling corresponding to dictated words and pictures in untaught sets. It seemed that there were two error patterns; auditory and visual discrimination between letters. Taro might not have enough ability to discriminate between the letters with common vowel (e.g., " $<$ " $(k u)$ and " $\supset$ " $(t s u))$ and between the letters with similar shape (e.g., “こ” (ko) and “(" $(i))$. The developmental level of speech and language would influence the spelling performance. Taro's speech and language development level was clearly lower than that of Ken and the participants of Greer et al. It would be necessary to consider the word sets to adjust discrimination ability of the participant with language developmental delay. However, Taro's performances in anagram spelling to printed word task were perfect in Sets 2 and 3. The strength of visualthinking in characteristics of autism spectrum disorders might influence these results.

Third, anagram spelling instruction might be ineffective in bringing up phonological awareness for Taro. This reasoning is supported because Taro displayed unstable vocal and anagram spelling for Sets 2 and 3 during the first probe (see Fig. 2). When individuals accomplish anagram spelling, phonological awareness is demanded (Dairoku, 1996). Thus, Taro's phonological awareness may not have been raised enough by the anagram spelling instruction. In addition, it is suggested that phonological awareness is usually developed at 4- to 5-year-old. Taro's verbal-social age was 2 years and 5 months and his vocabulary age was less than 3 years. On the other hand, Ken's mental and vocabulary age were ranged between $4-5$ years old. Taro did not have enough development of phonological awareness (e.g., moraic segmentation) because he segmented "tyo-da-i" word into not "tyo", "da", and " $\mathrm{i}$ " but "tyo" and "dai" before the anagram instruction. Further research is needed to investigate the relationship of anagram spelling and vocal spelling in an experiment where ability for phonological awareness and speech and language developmental age are controlled.

The present study has two more limitations. First, the internal validity is reduced because this study was conducted over an extended period of time and the intervention was only implemented once per week. The present study cannot deny the possibility that other variables influenced the improvement of the participants' spelling performances. In addition, the number of data points per phase was often rather limited. It was not sufficient to determine trend or variability of data and demonstrate experimental control. Horner, Carr, Halle, McGee, Odom, 
and Wolery (2005) noted that in most cases experimental control is demonstrated when the design documents three demonstrations of the experimental effects at three different points in time with a single participant (within-subject replication), or across different participants (inter-subject replication). Second, the external validity is also reduced because there were only two participants. Horner et al. noted that it is typical for single-subject studies to demonstrate effects with at least three different participants. It also is expected that experimental effects are replicated across participants, settings, or materials to establish external validity. The present study should increase the number of participants, and attempt to demonstrate the effects for individuals who have different learning histories. Future research should compare the result between the participants with controlled developmental age, and should discuss the participants' learning experience.

\section{Acknowledgment}

This work was supported by JSPS KAKENHI Grant Numbers JP15K17423, JP16H03790a8. The authors are grateful to Dr. Yoshihisa Ohtake (Okayama University) for his support.

\section{References}

Amano, K. (1977) On the formulation of the act of analyzing the syllabic structure of words and the learning of Japanese syllabic characters in moderately mentally retarded children. Japanese Journal of Educational Psychology, 25, 73-84. (in Japanese)

Dairoku, H. (1996) Learning process of reading Kana characters: Longitudinal study in individuals with developmental delay. Unpublished doctoral dissertation, Tokyo University. (in Japanese)

de Souza, A. A. \& Rehfeldt, R. A. (2013) Effects of dictationtaking and match-to-sample training on listing and spelling responses in adults with intellectual disabilities. Journal of Applied Behavior Analysis, 46, 792-804.

Greer, R. D., Yaun, L., \& Gautreaux, G. (2005) Novel dictation and intraverbal responses as a function of a multiple exemplar instruction instructional history. Analysis of Verbal Behavior, 21, 99-106.

Horner, R. H., Carr, E. G., Halle, J., McGee, G., Odom, S., \& Wol- ery, M. (2005) The use of single-subject research to identify evidence-based practice in special education. Exceptional Children, 71, 165-179.

Hubner, M. M. C., Gomes, R. C., \& McIlvane, W. J. (2009) Recombinative generalization in minimal verbal unit-based reading instruction for pre-reading children. Experimental Analysis of Human Behavior Bulletin, 27, 11-17.

Ikuzawa, M., Matsushita, Y., \& Nakase, A. (2002) Kyoto scale of psychological development 2001. Kyoto International Social Welfare Exchange Center, Kyoto. (in Japanese)

Kikuchi, E. (1985) Examination of the matching-to-sample procedure in the reading behavior of mentally retarded children. Japanese Journal of Special Education, 22, 20-30. (in Japanese)

Mackay, H. A. (1985) Stimulus equivalence in rudimentary reading and spelling. Analysis and Intervention in Developmental Disabilities, 5, 373-387.

Matos, M. A., Avanzi, A. L., \& McIlvane, W. J. (2006) Rudimentary reading repertoires via stimulus equivalence and recombination of minimal verbal units. Analysis of Verbal Behavior, 22, 3-19.

Morita, H., Nakayama, T., Sato, K., \& Maekawa, H. (1997) Help with acquisition on reading of Kana characters in dyslexic children. Japanese Journal of Learning Disabilities, 5, 49-62. (in Japanese)

Sakamoto, M., Hiruma, M., Hosokawa, M., Imanaka, M., \& Maekawa, H. (2004) Teaching Kana reading to a child with a specific difficulty with successive processing. Japanese Journal of Learning Disabilities, 13, 3-12. (in Japanese)

Sidman, M. (1971) Reading and auditory-visual equivalences. Journal of Speech and Hearing Research, 14, 5-13.

Sidman, M. \& Tailby, W. (1982) Conditional discrimination vs. matching to sample: An expansion of the testing paradigm. Journal of the Experimental Analysis of Behavior, 37, 5-22.

Sugasawara, H. \& Yamamoto, J. (2007) Computer-based teaching of word construction and reading in two students with developmental disabilities. Behavioral Interventions, 22, 263-277.

Tanaka Institute for Educational Research (2003). Tanaka-Binet Intelligence Scale-Fifth Edition. Taken Publishing, Tokyo. (in Japanese)

Tanji, T., Takahashi, K., \& Noro, F. (2013) Teaching generalized reading and spelling to children with autism. Research in Autism Spectrum Disorders, 7, 276-287.

Tsurumaki, M. (1995) Acquisition of textual behavior in children with mental retardation by a simultaneous visual-visual matching-to-sample procedure. Japanese Journal of Special Education, 32, 39-47. (in Japanese)

Ueno, K., Nagoshi, N., \& Konuki, S. (2008) Picture vocabulary test-revised. Nihon Bunka Kagakusha, Tokyo. (in Japanese) 